\title{
iPS, Organoids and 3D Models as Advanced Tools for In Vitro Toxicology
}

doi:10.14573/altex.1911071

Recent progress in the field of in vitro toxicology has led to the development of increasingly complex models that are closer to the complexity of organs and tissues, both in terms of structure and functionality, and are characterized by more precise, specific and early endpoints (Ranga et al., 2014; Xinaris et al., 2015; Clevers, 2016; Jackson and Lu, 2016; Bartfeld and Clevers, 2017; Pamies et al., 2018; Truskey, 2018). The transition from traditional 2D models to three-dimensional (3D) systems certainly represents the most important innovation of the last decades. Numerous studies now employ 3D systems, spheroids and organoids, also based on pluripotent stem cells, for the study of drug toxicity. However, the still high cost of these systems limits their use in xenobiotic screening and environmental toxicology (Kolaja, 2014; Gómez-Lechón and Tolosa, 2016; Luz and Tokar, 2018; Lynch et al., 2019).

The origin and development of advanced cellular models and the most recent and promising organoid techniques in the toxicological sector were discussed at the meeting "Organoids, iPS and 3D systems: advance models for in vitro toxicology" organized by CELLTOX Italian Association of in vitro Toxicology at Istituto di Ricerche Farmacologiche Mario Negri IRCCS (Milano, Italy) in May 2019. More than 100 people from academia, companies and research centers attended the event. The morning session was chaired by Diego Baderna, President of CellTox, and Susanna Alloisio, introducing the origin and the technological progress in the field of organoids and induced pluripotent stem cells (iPS).

The opening lecture was held by Nathalie Steimberg, Tissue Engineering Laboratory, Department of Clinical and Experimental Sciences at the University of Brescia, who introduced the advanced models from an historical point of view and the scientific background behind the enhanced complexity of in vitro models in the last 20 years. Used because of their simplicity, high reproducibility, and low cost, 2D cultures also have limited morpho-functional characteristics to correctly mimic organ features. 3D models allow cells to self-organize and polarize, to create their own extracellular matrix (ECM) as well as 3D interactions, adhesion and communication patterns with neighboring cells and surrounding ECM (Mazzoleni et al., 2009). Cells grown in 3D cultures better express their native functions, reaching more relevant physiological and tissue-specific characteristics. Tissue engineering-based strategies to optimize 3D models include the formation of spheroids or organoids in static or dynamic states, with or without scaffolds, and with biosignaling factors. The cells can be from healthy or pathological tissues/or- gans or from either terminally differentiated or pluripotent cells. Whereas spheroids can succinctly resemble tissues, it is generally accepted that, with a higher level of complexity and functionality, organoids better mimic organ behavior. In fact, thanks to spatiotemporally defined events (self-organization, lineage commitment, differentiation of progeny, as well as control of the spatially restricted cell-fate decision) and their ability to self-renew, stem cells in the presence of other cell types evolve into organ bud (Fatehullah et al., 2016; Lancaster and Knoblich, 2014). Organoids can recapitulate the gastrointestinal system, brain, heart, kidney, liver, lung, pancreas, prostate, etc. These models can be employed in various fields of application where physiological, pathological and developmental processes are studied to better translate basic research into clinical applications as well as to develop personalized therapeutics.

Manuela T. Raimondi, Politecnico di Milano, focused on frontier technologies for stem cells developed by her research group. The NICHOID technology is a substrate for the expansion of stem cells that mimics the micro-architectural cues of the physiological niche of stem cells (Raimondi et al., 2013, 2014; Nava et al., 2015, 2016, 2017). The substrate is polymerized with a femtosecond-pulsed laser in a biocompatible resin using two-photon laser polymerization (Raimondi et al., 2012; Ricci et al., 2017; Zandrini et al., 2019). NICHOID could drastically reduce the time to market of stem cell products, from currently 20 years to a few years. The miniaturized, optically-accessible perfusion bioreactor $(\mathrm{MOAB})$ allows culture of $3 \mathrm{D}$ organoids of up to a few millimeters in size under continuous perfusion of the culture medium. The test agent is infused and the cell response is assessed by fluorescence diagnostics, both in real time and post-cultivation (Laganà and Raimondi, 2012; Nava et al., 2013; Raimondi et al., 2015; Credi et al., 2016; Tunesi et al., 2016; Frattini et al., 2017; Marturano-Kruik et al., 2018; Izzo et al., 2019). The MOAB can accelerate the discovery of new drugs, including nanoparticle-based drugs and stem cell-based drugs. Further, the MICROATLAS miniaturized imaging window for intravital microscopy was presented, which is an implantable device to visualize and quantify the immune response to biomaterials, drugs and stem cell-based biodrugs in vivo over time. The MICROATLAS is less invasive than other technologies and can reduce the number of animals required by current regulation. The use of a 3D microstructured chip allows repositioning the observation field of view of a two-photon microscope for repeated and quantitative measurement of cell recruitment, neo-angiogenesis and fibrotic reactions at different time-points. 
Silvia Perego, Promega Italia, introduced industrial efforts to enhance the performance of cell-based assays applied to 3D systems. There is an increasing need for validated assays to measure markers in these culture models. Two basic factors to consider include the ability of the detection reagent to lyse all the cells forming the large 3D structures and the ability of small molecule detection probes to penetrate through the entire 3D structure. Working on reagents and protocols already applied to 2D culture models, Promega has developed a platform of bioluminescent and fluorescent cell-based assays suitable for 3D microtissues. Among the most biologically relevant markers, this platform includes assays for the measurement of ATP for cell viability, LDH for cytotoxicity, caspases for apoptosis, GSH for oxidative stress, and various metabolites for cell metabolism.

Alessia Bertero, Università degli Studi di Milano-DIMEVET, described promising 3D species-specific cultures as in vitro predictive tools for toxicological evaluations (Augustyniak et al., 2019). Species-specific organoids better mimic the in vivo tissue structure compared to 2D cultures, and they are flexible and characterized by genetic stability, thus their application as models in toxicological studies (i.e., in vitro evaluations of feed additives and environmental contaminants) could be beneficial. Species-specific organoids have been obtained from tissue-specific stem cells (SCs) of different sources: keratinocytes (canine), intestine (avian, bovine, canine and porcine), liver (canine and feline), mammary gland (bovine and caprine). Intestinal organoids, in particular porcine enteroids, have been recently applied as an in vitro predictive model in toxicology ( $\mathrm{Li}$ et al., 2019). Canine keratinocyte organoids, bovine mammary organoids, and canine and feline liver organoids could be suitable for target organ toxicity studies.

The afternoon session, chaired by Francesca Caloni and Isabella De Angelis, focused on organ-specific models.

Thomas Hartung, Center for Alternatives to Animal Testing (CAAT) and CAAT Europe, spoke about BrainSpheres, a 3D brain model derived from human-iPS cells. This technology allows the development of a reproducible neural model with respect to size and shape and including populations of neurons, astrocytes and oligodendrocytes (Pamies et al., 2017). It has been applied to study developmental neurotoxicity (Pamies et al., 2018) and is considered a promising tool for the study of neurodegenerative diseases (e.g., Parkinson's, ALS), infectious diseases (e.g., Zika, JC, Dengue) and for gene/environment interactions. Progress has been made in enhancing organ architecture and cell environment through the addition of microglia and coating with blood/brain-barrier. Glioblastoma tumor cells have been added into the spheroids to generate a 3D heterotypic glioblastoma brain sphere for screening anti-glioblastoma agents and evaluating personalized treatment options (Plummer et al., 2019).

The advantages of 3D bioprinting for the production of iPSCderived organoids of human neuronal tissue were introduced by Silvia Di Angelantonio, Sapienza University and IIT. Lack of appropriate in vitro models of the human nervous system hampers the development of effective drugs for neurological disor- ders. Conventional 2D cell cultures fail to represent the complexity of the brain and novel 3D systems are emerging as more realistic and representative models because they better reproduce the architecture of real organs and tumours in vitro. While not completely replacing animal experimentation, they provide valid pre-screening systems for potential drugs, reducing the number of candidate molecules to be tested in subsequent phases. To date, organoids from iPSCs have been generated by allowing them to self-organize into 3D structures during differentiation, generating varied structures in terms of size and internal architecture and affecting the reproducibility of the results. The technology developed in the HoMoLoG project aims to overcome this problem by $3 \mathrm{D}$ printing biological material (3D bioprinting) to produce iPSC-derived organoids in a more controlled and reproducible way. The procedure is based on the robotic manipulation of cellular material and organic encapsulation matrices, which are organized in space in a controlled manner, similar to the ink of a conventional 3D printer. A novel neural construct was generated by $3 \mathrm{D}$ bioprinting of cortical neurons and glial precursors derived from human iPSCs. Bioprinted cells are further differentiated within the construct and express neuronal and astrocytic markers. Functional analysis of 3D bioprinted cells highlights an early stage of maturation and the establishment of early network activity behavior (Salaris et al., 2019). This work lays the basis for generating more complex and faithful 3D models of the human nervous system to provide novel disease models of brain pathologies and to assess whether they can be used for drug testing and for developing new diagnostic tools.

Valeria Chiono, from DIMEAS (Politecnico di Torino) and Centro 3R, presented the preparation of biomimetic microenvironments for in vitro modelling of pathological cardiac tissue carried out within the BIORECAR project. After myocardial failure, the heart undergoes phenotypic changes with the progressive formation of a fibrotic scar (Porter and Turner, 2009). In vitro models of infarcted tissue represent a key tool to evaluate new therapies for cardiac regeneration, among which direct cardiac reprogramming is one of the most promising. To develop an in vitro model of cardiac tissue, the complexity of in vivo tissue physiology should be reproduced, such as cell adhesion, proliferation, and spatial alignment (Zhang et al., 2015). Preliminary unpublished data on direct cardiac reprogramming suggests that a $3 \mathrm{D}$ microenvironment may enhance conversion of human fibroblasts into cardiomyocytes, supporting the need for reliable 3D models of cardiac tissue. Such findings are in agreement with previous literature results on mouse fibroblast direct reprogramming ( $\mathrm{Li}$ et al., 2016). To better control and mimic biological features of infarcted tissue (e.g., cell-cell and cell-ECM interactions), a model of human cardiac fibroblasts (HCFs) cultured on 3D bioartificial scaffold was presented. Scaffolds were designed and fabricated with both aligned and random morphology by melt-extrusion additive manufacturing using a biocompatible synthetic polymer. SEM analysis demonstrated that 3D scaffolds had a regular and interconnected porous structure, with pore size ranging from 150 to 350 
$\mu \mathrm{m}$, depending on scaffold design. Scaffolds were also surface grafted with an adhesive protein to stimulate HCFs, isolated from human ventricle, to deposit extracellular matrix. In the future, the influence of scaffold properties (composition, structure and surface mechanical properties) on the expression of fibroblast markers ( $\alpha$-SMA, vimentin, discoidin domain receptor 2) and ECM composition will be evaluated. Once finalized, this model will represent a valuable tool for the in vitro testing of new cardiac regenerative strategies, including direct cardiac reprogramming.

Patrizia Dell'Era from the Cellular Fate Reprogramming Unit (FRU lab) at the University of Brescia, focused on the use of iPSC-derived cardiac and hepatic cells in toxicological studies. Induced iPS derived cells have become one the most valuable tools to fulfil the European $3 \mathrm{R}$ request to reduce, refine, and replace animal testing. In the FRU lab, work with human iPSCs to model atrial fibrillation started in 2011. Thus, iPS-derived human cardiomyocytes have been obtained and characterized. However, the biological properties of these spontaneously contractile cells point to a phenotype that is far from their adult counterpart: The cells exhibit a fetal-like phenotype, showing the contemporary expression of the fetal and the mature forms of cardiac troponin I, as well as a higher expression of embryonal myosin heavy and light chains. Their action potential was traced but, again, their firing is far different from an adult human cardiomyocyte. Moreover, the culture is a mix of atrial, ventricular and nodal cardiomyocytes, raising doubt on their use in toxicological studies (Barbuti et al., 2016). Another example involves the generation of human hepatocytes from iPSC. Even though the data are very preliminary, iPSC-derived hepatocytes obtained in the FRU lab share the same immaturity as the cardiomyocytes. Indeed, after 20 days of differentiation, a decrease in alpha-fetoprotein paralleled by an initial increase in albumin production can be observed. A similar result was also obtained using hepatic organoids, where iPSC-derived hepatocytes were assembled with iPSC-derived mesenchymal stem cells and endothelial cells in a 3D structure. In conclusion, iPSC-derived cardiac and hepatic cells can be reliable models for toxicological studies, but the immaturity of these cells and the challenges of dealing with mixed cell populations must be taken into account for toxicology experiments.

Recent success with engineering human organoids and tissues has created great expectations, especially regarding their potential as tissue substitutes. The most feasible current applications for organoid technologies are drug testing, disease modelling, and studying human development. In his lecture, Christodolous Xinaris, from Istituto Mario Negri, discussed current and future applications of tissue engineering and organoids. He described the newly developed platform for growing functional mouse and human chimeric kidney organoids in vivo from embryonic kidney cells and analyzed an example of combining engineering and 3D technologies to generate patient-specific kidney tissue for modelling human kidney disease, studying human kidney development, and discovering new drugs. Moreover, Dr Xinaris presented the potential applications of iPSC-derived tissues in regenerative medicine through an experimental approach to curing anemia.

Taken together, 3D cell cultures are able to increase the ability of in vitro models to mimic real physiological conditions, making the system more representative and informative. At the same time, by allowing preliminary laboratory analyses on organoids derived from patients, this technology represents a promising tool for personalized medicine, leading to potential advantages in terms of drug efficacy and patient safety. However, there are some critical issues to consider when approaching this technology for toxicological screening, such as the reproducibility of the model, the availability of the biomaterials, and the selection of assays and probes specific for these advanced systems. The workshop showed that important progress has been made to make 3D models increasingly reliable, reproducible, economical, and accessible to a wider audience.

\section{References}

Augustyniak, J., Bertero, A., Coccini, T. et al. (2019). Organoids are promising tools for species-specific in vitro toxicological studies. J Appl Toxicol 39, 1610-1622. doi:10.1002/jat.3815

Barbuti, A., Benzoni, P., Campostrini, G. et al. (2016). Human derived cardiomyocytes: A decade of knowledge after the discovery of induced pluripotent stem cells. Dev Dyn 245, 1145 1158. doi:10.1002/dvdy. 24455

Bartfeld, S. and Clevers, H. (2017). Stem cell-derived organoids and their application for medical research and patient treatment. J Mol Med 95, 729-738. doi:10.1007/s00109-0171531-7

Clevers, H. (2016). Modeling development and disease with organoids. Cell 165, 1586-1597. doi:10.1016/j.cell.2016.05.082

Credi, C., De Marco, C., Molena, E. et al. (2016). Direct photo-patterning of hyaluronic acid baits onto a fouling-release perfluoropolyether surface for selective cancer cells capture and immobilization. Mater Sci Eng C Mater Biol Appl 62, 414-422. doi:10.1016/j.msec.2015.12.063

Fatehullah, A., Tan, S. H., Barker, N. (2016). Organoids as an in vitro model of human development and disease. Nat Cell Biol 18, 246-254. doi:10.1038/ncb3312

Frattini, P., Villa, C., De Santis, F. et al. (2017). Autologous intramuscular transplantation of engineered satellite cells induces exosome-mediated systemic expression of Fukutin-related protein and rescues disease phenotype in a murine model of limb-girdle muscular dystrophy type 2I. Hum Mol Genet 26 , 3682-3698. doi:10.1093/hmg/ddx252

Gómez-Lechón, M. J. and Tolosa, L. (2016). Human hepatocytes derived from pluripotent stem cells: A promising cell model for drug hepatotoxicity screening. Arch Toxicol 90, 20492061. doi:10.1007/s00204-016-1756-1

Izzo, L., Tunesi, M., Boeri, L. et al. (2019). Influence of the static magnetic field on cell response in a miniaturized optically accessible bioreactor for 3D cell culture. Biomed Microdevices 21, 29. doi:10.1007/s10544-019-0387-8 
Jackson, E. L. and Lu, H. (2016). Three-dimensional models for studying development and disease: Moving on from organisms to organs-on-a-chip and organoids. Integr Biol (Camb) 8 , 672-683. doi:10.1039/C6IB00039H

Kolaja, K. (2014). Stem cells and stem cell-derived tissues and their use in safety assessment. J Biol Chem 289, 4555-4561. doi:10.1074/jbc.R113.481028

Laganà, M. and Raimondi, M. T. (2012). A miniaturized, optically accessible bioreactor for systematic 3D tissue engineering research. Biomed Microdevices 14, 225-234. doi:10.1007/ s10544-011-9600-0

Lancaster, M. A. and Knoblich, J. A. (2014). Organogenesis in a dish: Modeling development and disease using organoid technologies. Science 345, 1247125. doi:10.1126/science. 1247125

Li, X. G., Zhu, M., Chen, M. X. et al. (2019). Acute exposure to deoxynivalenol inhibits porcine enteroid activity via suppression of the Wnt/ $\beta$-catenin pathway. Toxicol Lett 305, 19-31. doi:10.1016/j.toxlet.2019.01.008

Li, Y., Dal-Pra, S., Mirotsou, M. et al. (2016). Tissue-engineered 3-dimensional (3D) microenvironment enhances the direct reprogramming of fibroblasts into cardiomyocytes by microRNAs. Sci Rep. 6, 38815. doi:10.1038/srep38815

Luz,A. L. and Tokar, E. J.(2018). Pluripotent stem cells in developmental toxicity testing: A review of methodological advances. Toxicol Sci 165, 31-39. doi:10.1093/toxsci/kfy174

Lynch, S., Pridgeon, C. S., Duckworth, C. A. et al. (2019). Stem cell models as an in vitro model for predictive toxicology. Biochem J 476, 1149-1158. doi:10.1042/BCJ20170780

Marturano-Kruik, A., Nava, M. M., Yaeger, K. et al. (2018). Human bone perivascular niche-on-a-chip for studying metastatic colonization. Proc Natl Acad Sci U S A 115,1256-1261. doi:10.1073/pnas.1714282115

Mazzoleni, G., Di Lorenzo, D. and Steimberg, N. (2009). Modelling tissues in 3D: The next future of pharmaco-toxicology and food research? Genes Nutr 4, 13-22. doi:10.1007/s12263008-0107-0

Nava, M. M., Raimondi, M. T. and Pietrabissa, R.A. (2013). Multiphysics 3D model of tissue growth under interstitial perfusion in a tissue-engineering bioreactor. Biomech Model Mechanobiol 12, 1169-1179. doi:10.1007/s10237-013-0473-4

Nava, M. M., Raimondi, M. T., Credi, C. et al. (2015). Interactions between structural and chemical biomimetism in synthetic stem cell niches. Biomed Mater 10, 015012. doi:10.1088/1748-6041/10/1/015012

Nava, M. M., Piuma, A., Figliuzzi, M. et al. (2016). Two-photon polymerized "nichoid" substrates maintain function of pluripotent stem cells when expanded under feeder-free conditions. Stem Cell Res Ther 7, 132. doi:10.1186/s13287-016-0387-z

Nava, M. M., Di Maggio, N., Zandrini, T. et al. (2017). Synthetic niche substrates engineered via two-photon laser polymerization for the expansion of human mesenchymal stromal cells. J Tissue Eng Reg Med 11, 2836-2845. doi:10.1002/term.2187

Pamies, D., Barreras, P., Block, K. et al. (2017). A human brain microphysiological system derived from induced pluripo- tent stem cells to study neurological diseases and toxicity. ALTEX 34, 362-376. doi:10.14573/altex.1609122

Pamies, D., Bal-Price, A., Chesné, C. et al. (2018). Advanced good cell culture practice for human primary, stem cell-derived and organoid models as well as microphysiological systems. ALTEX 35, 353-378. doi:10.14573/altex.1710081

Plummer, S., Wallace, S., Ball, G. et al. (2019). A human iPSC-derived 3D platform using primary brain cancer cells to study drug development and personalized medicine. Sci Rep 9, 1407. doi:10.1038/s41598-018-38130-0

Porter, K. E., Turner, N. A. (2009). Cardiac fibroblasts: At the heart of myocardial remodeling. Pharmacol Ther 123, 255278. doi:10.1016/j.pharmthera.2009.05.002

Raimondi, M. T., Eaton, S. M., Nava, M. M. et al. (2012). Two-photon laser polymerization: From fundamentals to biomedical application in tissue engineering and regenerative medicine. J Appl Biomater Function Mater 10, 55-65. doi:10.5301/JABFM.2012.9278

Raimondi, M. T., Eaton, S. M., Laganà M. et al. (2013). Three-dimensional structural niches engineered via two-photon laser polymerization promote stem cell homing. Acta Biomater 9, 4579-4584. doi:10.1016/j.actbio.2012.08.022

Raimondi, M. T., Nava, M. M., Eaton, S. M. et al. (2014). Optimization of femtosecond laser polymerized structural niches to control mesenchymal stromal cell fate in culture. Micromachines 5, 341-358. doi:10.3390/mi5020341

Raimondi, M. T., Giordano, C. and Pietrabissa, R. (2015). Oxygen measurement in interstitially-perfused cellularised constructs cultured in a miniaturized bioreactor. J Appl Biomater Funct Mater 13, e313-e319. doi:10.5301/jabfm.5000246

Ranga, A., Gjorevski, N. and Lutolf, M. P. (2014). Drug discovery through stem cell-based organoid models. Adv Drug Deliv Rev 69-70, 19-28. doi:10.1016/j.addr.2014.02.006

Ricci, D., Nava, M. M., Zandrini, T. et al. (2017). Scaling-up techniques for the nanofabrication of cell culture substrates via two-photo polymerization for industrial-scale expansion of stem cells. Materials 10, 66. doi:10.3390/ma10010066

Salaris, F., Colosi, C., Brighi, C. et al. (2019). 3D bioprinted human cortical neural constructs derived from induced pluripotent stem cells. J Clin Med 8, 1595. doi:10.3390/jcm8101595

Truskey, G. A. (2018). Human microphysiological systems and organoids as in vitro models for toxicological studies. Front Public Health 6, 185. doi:10.3389/fpubh.2018.00185

Tunesi, M., Fusco, F., Fiordaliso, F. et al. (2016). Optimization of a $3 \mathrm{D}$ dynamic culturing system for in vitro modeling of frontotemporal neurodegeneration-relevant pathologic features. Front Aging Neurosci 8, 146. doi:10.3389/fnagi.2016.00146

Xinaris, C., Brizi, V. and Remuzzi, G. (2015). Organoid models and applications in biomedical research. Nephron 130, 191199. doi:10.1159/000433566

Zandrini, T., Shan, O., Parodi, V. et al. (2019). Multi-foci laser microfabrication of 3D polymeric scaffolds for stem cell expansion in regenerative medicine. Sci Rep 9, 11761. doi:10.1038/s41598-019-48080-w 
Zhang, Y. S., Aleman, J., Arneri, A. et al. (2015). From cardiac tissue engineering to heart-on-a-chip: Beating challenges. Biomed Mater Bristol Engl 10, 034006. doi:10.1088/17486041/10/3/034006

\section{Acknowledgments}

The work presented by Prof. V. Chiono was supported by BIORECAR project (financed by European Research Council (ERC) under the European Union's Horizon 2020 research and innovation programme; grant agreement No 772168). The projects MOAB (grant agreement No 825159), NICHOID (grant agreement No 646990) and NICHOIDS (grant agreement No 754467) received funding from the European Research Council (ERC) under the EU Horizon 2020 research and innovation programme. The project BEYOND (No R16ZNN2R9K) has received funding from the Italian Ministry of University and Research (MIUR) under the grant program MIUR-FARE-2016.
Nathalie Steimberg ${ }^{1}$, Alessia Bertero ${ }^{2}$, Valeria Chiono $^{3}$, Patrizia Dell'Eral', Silvia Di Angelantonio ${ }^{4,5}$,

Thomas Hartung ${ }^{6}$, Silvia Perego ${ }^{7}$, Manuela Raimondi ${ }^{8}$, Christodoulos Xinaris ${ }^{9}$, Francesca Caloni ${ }^{2}$, Isabella De Angelis ${ }^{10}$, Susanna Alloisio ${ }^{11,12}$ and Diego Baderna ${ }^{13}$

${ }^{1}$ Università degli Studi di Brescia, Brescia, Italy; ${ }^{2}$ Università degli Studi di Milano, Milan, Italy; ${ }^{3}$ Politecnico di Torino, Turin, Italy; ${ }^{4}$ Department of Physiology and Pharmacology, Sapienza University, Rome, Italy;

${ }^{5}$ Center for Life Nanoscience, Istituto Italiano di Tecnologia, Rome, Italy;

${ }^{6}$ Johns Hopkins Bloomberg School of Public Health, Baltimore, MD, USA; ${ }^{7}$ Promega Italia, Milan, Italy; ${ }^{8}$ Department of Chemistry, Materials and Chemical Engineering "G. Natta”, Politecnico di Milano, Milan, Italy; ${ }^{9}$ Laboratory of Organ Regeneration, Istituto di Ricerche Farmacologiche Mario Negri IRCCS, Milan, Italy; ${ }^{10}$ Istituto Superiore di Sanità, Rome, Italy; ${ }^{11}$ E.T.T. solutions, Genoa, Italy; ${ }^{12}$ Institute of Biophysics, National Research Council, Genova, Italy; ${ }^{13}$ Laboratory of Environmental Chemistry and Toxicology, Istituto di Ricerche Farmacologiche Mario Negri IRCCS, Milan, Italy

\section{Meeting Report}

\section{Animalfree Research Forum 2019: Animal-free Education}

doi:10.14573/altex.1912091

Animalfree Research is a foundation based in Switzerland that supports the research and implementation of the 3Rs principles, with a focus on the replacement of animal experiments. The foundation strives to distribute knowledge about alternative methods and seeks an open and respectful dialogue between the different stakeholders. Every year, Animalfree Research organizes a forum to discuss a topic of high scientific and societal relevance among researchers, students, policymakers, and the interested general public. The $9^{\text {th }}$ Animalfree Research Forum was held on October 31, 2019 in Zurich, Switzerland on “Animal-free Education” (Fig. 1).

Dr Silvia Frey (Executive Director, Animalfree Research) opened the Forum with an outline of the foundation's main working areas such as research funding, policy work, and education. She highlighted the fact that although the number of animals used for educational purposes is much lower than for example in basic scientific research, the real numbers are certainly higher in Switzerland than the reported statistical figures as the Swiss animal experiment statistics only represent those animals that are protected by animal protection legislation. In Switzerland, with a few exceptions for invertebrates, this legislation only considers vertebrates. Four presentations by internal and external speakers followed.

In the first talk, Dr Miriam Zemanova (Scientific Associate, Animalfree Research) introduced the background of the Forum

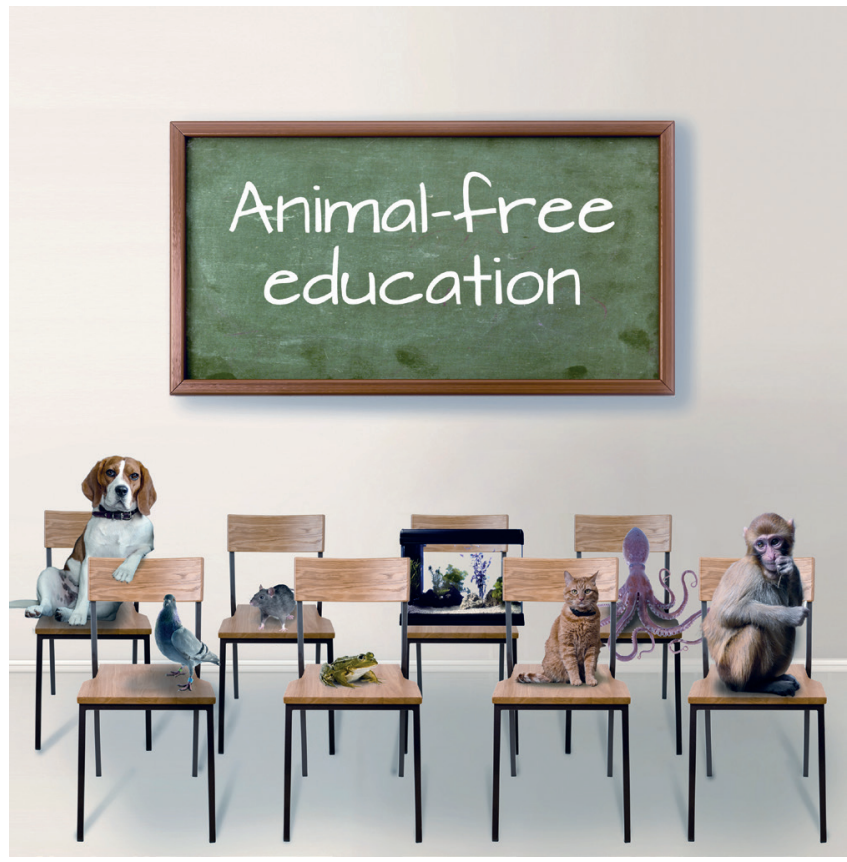

Fig. 1: A leaflet announcing the topic of the Forum 